\title{
Direct Precipitation and Characterization of ZnO Nanoparticles
}

\author{
A. H. Moharram, ${ }^{1}$ S. A. Mansour, ${ }^{1}$ M. A. Hussein, ${ }^{1,2}$ and M. Rashad $^{3,4}$ \\ ${ }^{1}$ Physics Department, College of Science and Arts, King Abdulaziz University, Rabigh 21911, Saudi Arabia \\ ${ }^{2}$ Physics Department, Faculty of Science, Zagazig University, Zagazig 44519, Egypt \\ ${ }^{3}$ Physics Department, Faculty of Science, Assiut University, Assiut 71516, Egypt \\ ${ }^{4}$ Physics Department, Faculty of Science, Tabuk University, P.O. Box 741, Tabuk 71491, Saudi Arabia
}

Correspondence should be addressed to A. H. Moharram; mohar200@yahoo.com

Received 12 September 2013; Accepted 26 December 2013; Published 6 February 2014

Academic Editor: Bin Zhang

Copyright (C) 2014 A. H. Moharram et al. This is an open access article distributed under the Creative Commons Attribution License, which permits unrestricted use, distribution, and reproduction in any medium, provided the original work is properly cited.

\begin{abstract}
$\mathrm{ZnO}$ nanoparticles are prepared through hydrolysis and condensation of zinc acetate dihydrate by potassium hydroxide in alcoholic medium at low temperatures. Thermal gravimetric analysis (TGA) of the precursor is made in order to specify the temperature range over which the weight loss and thermal effect are significant. X-ray diffraction of the as-prepared specimens shows that the hexagonal ( $a=3.2459 \AA, c=5.1999 \AA)$ structure is the predominant crystallographic structure. According to Scherer's formula, the average size of the nanoparticles is $22.4 \pm 0.6 \mathrm{~nm}$. The structural properties of the synthesized $\mathrm{ZnO}$ nanoparticles have been confirmed using the TEM micrographs. The optical energy gap of the $\mathrm{ZnO}$ nanoparticles, as obtained from applying Tauc's equation, is equal to $3.52 \mathrm{eV}$, which is higher than that of the bulk material. Absorption peak of the as-prepared sample is $298 \mathrm{~nm}$ which is highly blue shifted as compared to the bulk $(360 \mathrm{~nm})$. Large optical energy gap and highly blue shifted absorption edge confirm that the prepared $\mathrm{ZnO}$ nanoparticle exhibits strong quantum confinement effect.
\end{abstract}

\section{Introduction}

Semiconductor zinc oxide $(\mathrm{ZnO})$ nanoparticles have attracted much attention because of their interest in fundamental study and also their applied aspects such as in solar energy conversion, varistors, luminescence, photocatalysis, electrostatic dissipative coating, transparent UV protection films, and chemical sensors [1-4]. Various methods such as thermal decomposition, chemical vapor deposition, sol gel, spray pyrolysis, and precipitation have been developed for the fabrication of nanosized $\mathrm{ZnO}$ particles with uniform morphology and size [5-9]. Among these synthetic routes, precipitation approach compared with other traditional methods provides a facile way for low cost and large-scale production, which does not need expensive raw materials and complicated equipments [10].

$\mathrm{ZnO}$ nanoparticles have been synthesized by Han et al. [11] via precipitation-pyrolysis $(\mathrm{P} \& \mathrm{P})$, where the precursor zinc hydroxide carbonate $\left(\mathrm{Zn}_{5}\left(\mathrm{CO}_{3}\right)_{2}(\mathrm{OH})_{6}\right)$ was obtained. Their TEM results indicated that pyrolysis temperature is the predominant factor for controlling the mean size of nanoparticles, ranging from $8 \mathrm{~nm}$ to $80 \mathrm{~nm}$. It was found that increasing the pyrolysis temperature enhances the mean size. Highly dispersed uniform $\mathrm{ZnO}$ particles of different sizes and shapes were prepared by slowly adding zinc salt and sodium hydroxide solutions in parallel into aqueous solutions of Arabic gum [12]. Except for the very early stages, the precipitated solids consisted of a well-defined zinc oxide phase. The reaction temperature affected both the size of the precursors and their arrangement in the final particles. At ambient temperature the primary nanoparticles, approximately $10 \mathrm{~nm}$ in size, formed spherical aggregates, while at $600^{\circ} \mathrm{C}$ they were much larger $(44 \mathrm{~nm})$ and combined to form rather uniform hexagonal $\mathrm{ZnO}$ prisms.

In the present work, a direct precipitation method is employed to synthesize the nanosized $\mathrm{ZnO}$ particles using the raw materials. Characterization of the obtained particles has been made using X-ray diffractometry in addition to the particles morphology using the transmission electron microscope. To get more information about the synthesized 
particles, the optical properties of the resulted colloidal solution are investigated using UV-VIS double beam spectrophotometer.

\section{Experimental Techniques}

$\mathrm{ZnO}$ nanoparticles are prepared using the method which was reported previously by Pacholski et al. [13] through hydrolysis and condensation of zinc acetate dihydrate by potassium hydroxide in alcoholic medium at low temperatures. $\mathrm{ZnO}$ nanoparticles are settled at the bottom and the excess mother liquor was removed; then the precipitate was washed several times with methanol. The energy dispersive spectrometry (EDS) analysis for detecting the characteristic X-rays of the constituent elements is carried out using an X-Max 80 detector unit which was equipped with transmission electron microscope (TEM) JEM-1230.

Thermal gravimetric analysis (TGA) was carried out using a simultaneous DTA-TG apparatus (DTG-60H, Shimadzu Co., Japan). Approximately $20 \mathrm{mg}$ of the sample is placed in a platinum crucible on the pan of the microbalance and heated from room temperature to $700^{\circ} \mathrm{C}$, using $\mathrm{Al}_{2} \mathrm{O}_{3}$ as inert material. Analysis was performed under nitrogen flow at heating rate of $10^{\circ} \mathrm{C} / \mathrm{min}^{-1}$. Powder XRD measurements were performed using the X-ray diffractometer (Shimadzu $\mathrm{XD}-3 \mathrm{~A})$ in the diffraction angle range $20 \leq 2 \theta \leq 80^{\circ}$, with monochromatic $\mathrm{CuK} \alpha$ radiation $(\lambda=1.5418 \AA)$ source. High-resolution transmission electron microscope (HRTEM, JEM 2100) was used with a high resolution pole piece operates at $200 \mathrm{kV}$ accelerating voltage. It works under a vacuum of $\sim 10^{-6} \mathrm{~Pa}$. These conditions give a lattice resolution of $0.14 \mathrm{~nm}$ and a point to point resolution of $0.23 \mathrm{~nm}$ which help the instrument to be a perfect technique for imaging materials on the atomic scale. The optical properties of the nanoparticles in solutions are studied using UV-Visible spectrophotometer (Shimadzu, UV-2450) in the wavelengths range of 200$900 \mathrm{~nm}$.

\section{Results and Discussion}

After washing the synthesized material in methanol and drying with air, it shows a powdered form with white color. The purity of the as-prepared nanoscale $\mathrm{ZnO}$ particles was further confirmed by the energy dispersive X-ray spectroscopy (EDS). The EDS spectrum indicates the presence of only $\mathrm{Zn}$ and $\mathrm{O}$ elements in 1:1 atomic ratio in the analyzed $\mathrm{ZnO}$ sample. In order to understand the phase symmetry of the synthesized $\mathrm{ZnO}$ nanocrystals, a systematic study on the XRD was performed. The weight loss of the investigated material was analyzed. Figure 1 shows the thermogravimetric analysis (TGA) of the synthesized nanoparticles. In case of the as-prepared $\mathrm{ZnO} \mathrm{NPs}$, high rate of mass loss has occurred in the temperature range $30^{\circ} \mathrm{C} \leq T \leq 100^{\circ} \mathrm{C}$ due to the evaporation of the water absorbed on the surface of the products. At higher temperatures, weight loss continues up to $500^{\circ} \mathrm{C}$, which is not the case for the calcinated $\mathrm{ZnO}$ NPs. In the latter specimens, the mass loss ends at about $300^{\circ} \mathrm{C}$ which is due to the decomposition of $\mathrm{Zn}(\mathrm{OH})_{2}$. Comparison

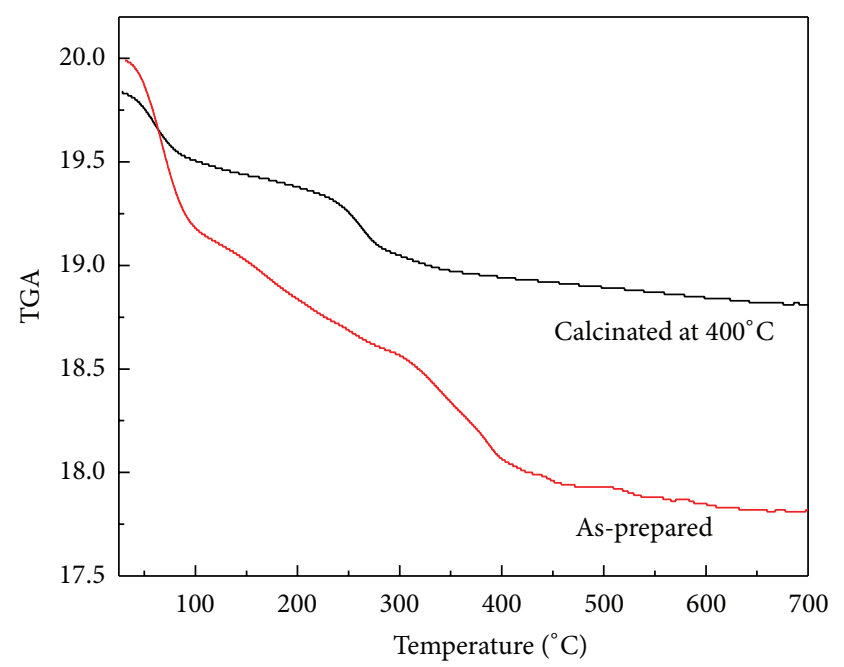

FIGURE 1: The thermogravimetric analysis (TGA) of the synthesized $\mathrm{ZnO}$ nanoparticles.

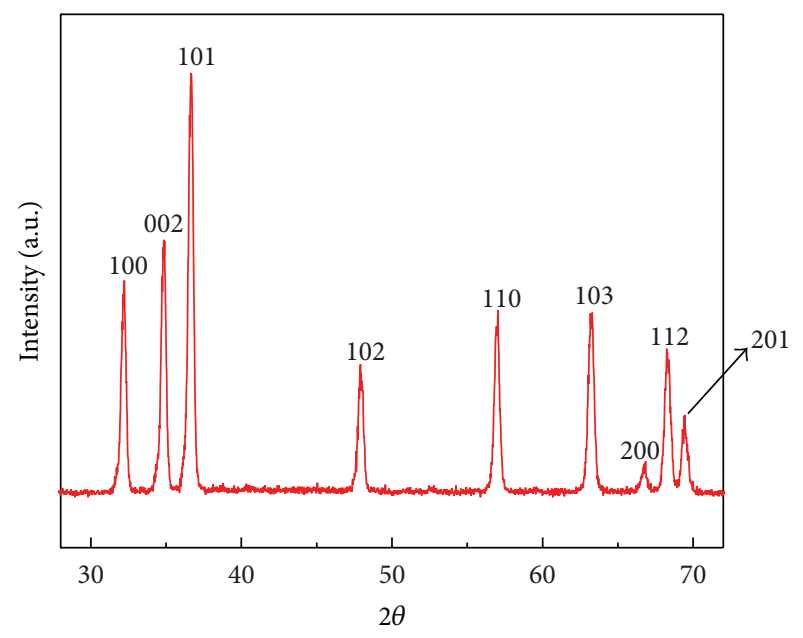

FIGURE 2: X-ray diffraction pattern of the synthesized $\mathrm{ZnO}$ nanoparticles.

between these two TGA curves, shown in Figure 1, indicates the quality improvement of the ZnO NPs after calcination process at $400^{\circ} \mathrm{C}$.

Figure 2 shows the XRD pattern for the synthesized $\mathrm{ZnO}$ nanoparticles. No extra diffraction peaks corresponding to $\mathrm{Zn}$, or other $\mathrm{ZnO}$ phases are detected, indicating that the pure $\mathrm{ZnO}$ nanoparticles are crystalline in nature. The peaks' intensity is sharp and narrow, confirming that the sample is of high quality with good crystallinity and fine grain size. XRD pattern of the as-prepared specimen shows that the hexagonal ( $a=3.2459 \AA, c=5.1999 \AA$ ) structure is the predominant crystallographic structure according to the Cross-Ref PDF number 04-015-4060 card [14]. Typical XRD pattern for hexagonal structure shows three strongest lines at $2 \theta$ values equal to $31.76^{\circ}, 34.58^{\circ}$, and $36.67^{\circ}$ due to reflection from the crystallographic (100), (002), and (101) planes, respectively. Other reflection peaks have been summarized in Table 1 . 
TABLE 1: X-ray reflection planes of the synthesized ZnO NPs obtained at different diffraction angles $(2 \theta)$ and the corresponding values of the maximum width at half maximum $(\beta)$.

\begin{tabular}{lcc}
\hline Planes (hkl) & $2 \theta^{\circ}$ & $\beta^{\circ}$ \\
\hline 100 & 31.76 & 0.384 \\
002 & 34.58 & 0.381 \\
101 & 36.68 & 0.39 \\
102 & 47.9 & 0.40 \\
110 & 57.02 & 0.41 \\
103 & 63.3 & 0.449 \\
200 & 66.68 & 0.39 \\
112 & 68.26 & 0.46 \\
201 & 69.36 & 0.45 \\
\hline
\end{tabular}

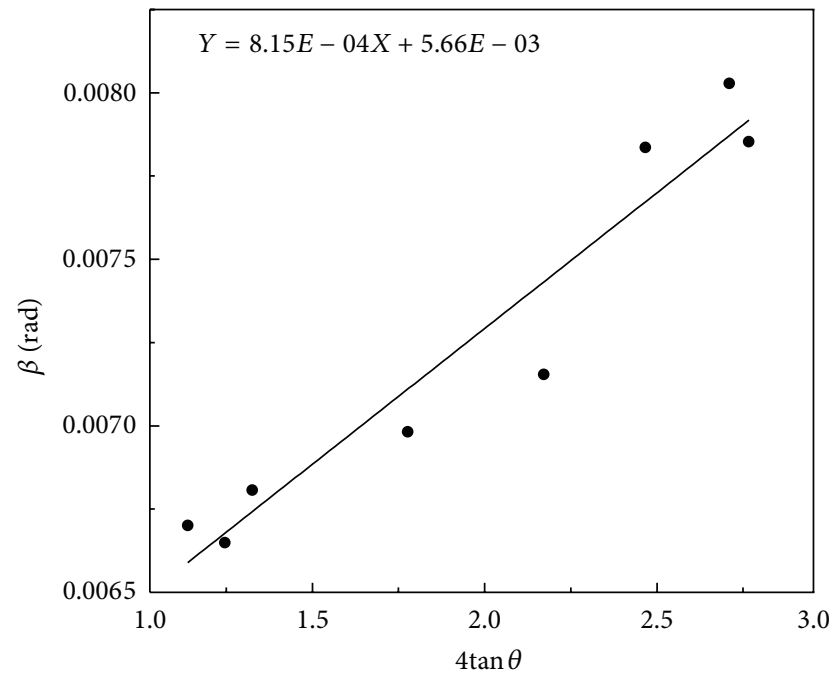

Figure 3: Plot of the full width at half maximum ( $\beta$ in radians) versus $4 \tan \theta$ of the synthesized $\mathrm{ZnO}$ nanoparticles.

The average size of the nanocrystals is estimated according to the Debye-Scherer formula [15]:

$$
D=\frac{K \lambda}{\beta \cos \theta},
$$

where the constant $K$ is taken to be $0.94, \lambda$ is the wavelength of the $\mathrm{CuK} \alpha$ radiation source, and $\beta$ the full width at half maximum (FWHM-in radians) of the diffraction peak corresponding to the Bragg diffraction angle $(2 \theta)$. Using the above equation, the average crystallite size is found to be in the range of $22.4 \pm 0.6 \mathrm{~nm}$. The strain induced in the obtained material due to crystal imperfection and distortion was calculated [16] using the formula:

$$
\varepsilon=\frac{\beta}{4 \tan \theta} .
$$

According to the above equation, plotting the values of $\beta$ (in $\operatorname{rad)~versus~} 4 \tan \theta$ should give a straight line, as shown in Figure 3. From the least square fit to the experimental points, the slope of the straight line gives directly the $\varepsilon$-value $\left(8.15 \times 10^{-4}\right)$ of the $\mathrm{ZnO}$ nanoparticles. In a previous work [17], the internal lattice strain value was found to be $14.6 \times$ $10^{-4}$ for the $\mathrm{ZnO}$ nanoparticles. A value which is higher than the present one indicates that the as-prepared $\mathrm{ZnO}$ nanoparticles have less imperfections than those reported in [17]. These strain values confirm the specimen uniformity in all crystallographic directions, thus considering the isotropic nature of the crystal, where the material properties are independent of the direction along which they are measured.

The TEM morphology of the synthesized $\mathrm{ZnO}$ nanoparticles is shown in Figure 4. The mean size estimated from the TEM image is about $20 \pm 0.5 \mathrm{~nm}$ and clearly indicates that the $\mathrm{ZnO}$ nanoparticles are crystalline with a wurtzite structure. This is in close agreement with the results calculated from powder XRD data using the Debye-Scherrer formula.

To characterize the optical properties of the obtained specimens, solutions of the synthesized nanoparticles have been made. While it is slightly soluble in methanol, it shows a stable colloid in mixture of methanol and chloroform [14]. The spectral dependence of the absorbance is measured using UV-VIS double beam spectrophotometer in the wavelength range $300-2500 \mathrm{~nm}$. Figure 5 shows the variations of the absorption coefficient with the energy of the incident photons. The absorption coefficient increases slowly with increasing the wavelength of the incident photons up to a certain value after which it increases very rapidly showing a peak at about $298 \mathrm{~nm}$. It is well known that a narrow absorption peak means a good crystalline specimen. The absorption peak located at $298 \mathrm{~nm}$ in case of the obtained nanoparticles is highly blue shifted as compared with the bulk (360 nm) material. In the high absorption region $\left(\alpha \geq 10^{4} \mathrm{~cm}^{-1}\right)$, the spectral dependence of the absorption coefficient can be described by Tauc relation:

$$
\alpha=\frac{c}{h \nu}\left(h v-E_{o}\right)^{1 / 2} .
$$

Taking the square of the above equation gives

$$
(\alpha h v)^{2}=c\left(h v-E_{o}\right),
$$

where $c$ is a constant of proportionality and $E_{o}$ is optical energy gap of the investigated solution. The experimental points fit with the above equation only if the direct electronic transitions are responsible for the photon absorption inside the nanoparticles. Intercept of the straight line with the photon energy axis at $(\alpha h v)^{2}=0$, as shown in Figure 6, yields the optical energy gap $(=3.52 \mathrm{eV})$, a value which is in good agreement with that $(=3.53 \mathrm{eV})$ previously obtained [17], while it is slightly higher than that $(3.4 \mathrm{eV})$ previously reported by Samuel et al. [18]. Band gap energy increases with decreasing particle size due to quantum size effects.

\section{Conclusions}

The structural and optical properties of the synthesized $\mathrm{ZnO}$ nanoparticles have been confirmed using TEM, XRD, and UV-VIS spectroscopy. According to Scherer's formula, the average particle size of the sample is $22.4 \pm 0.6 \mathrm{~nm}$. The optical energy gap of the $\mathrm{ZnO}$ nanoparticles, as obtained 

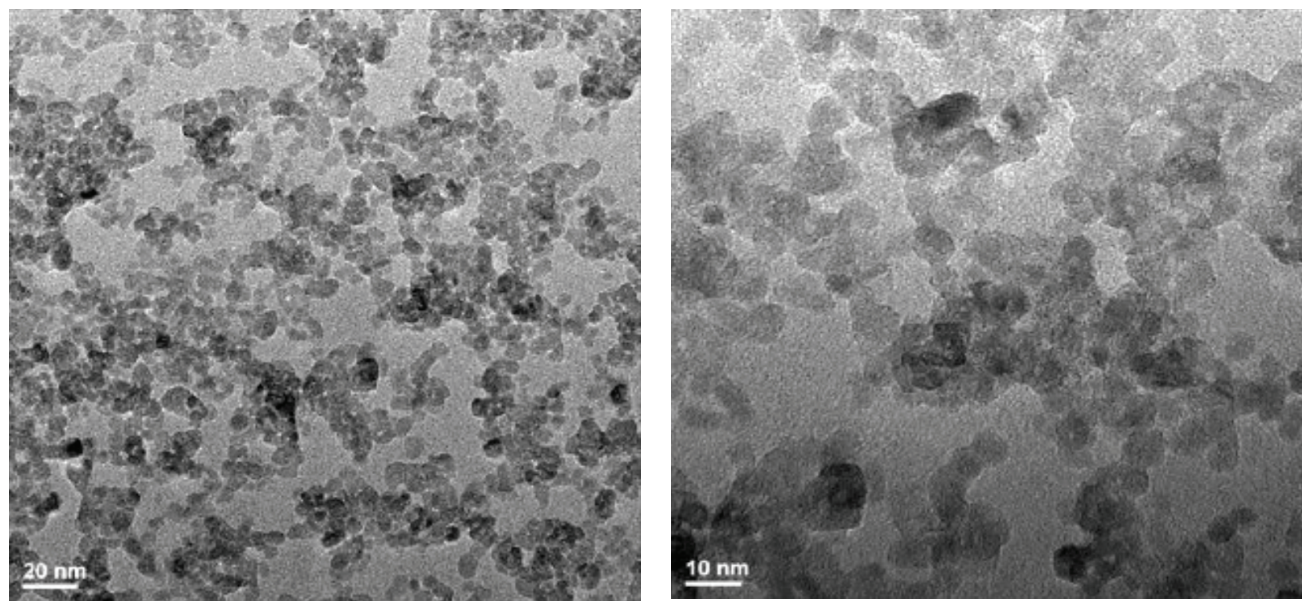

FIGURE 4: TEM micrograph of the synthesized $\mathrm{ZnO}$ nanoparticles.

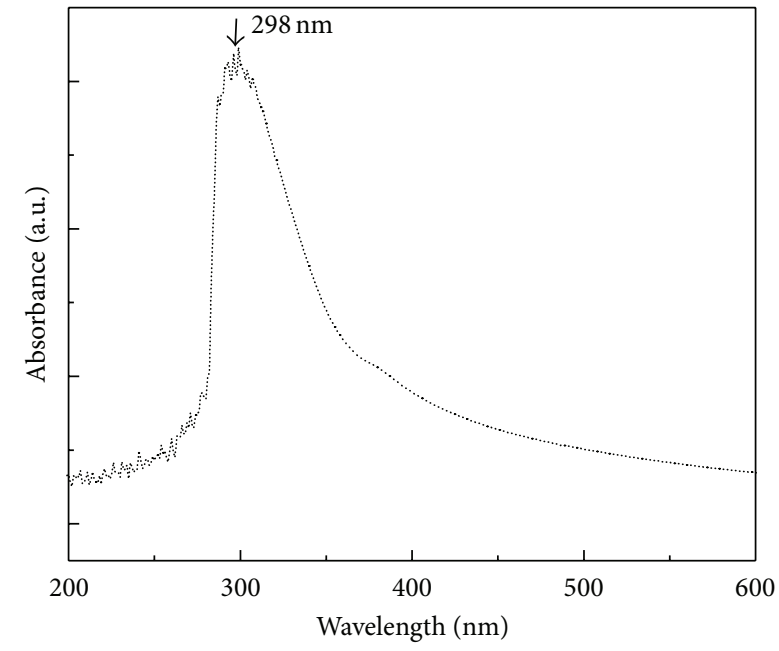

FIGURE 5: The dependence of the absorption coefficient on the wavelength of the incident photons through solution of the synthesized $\mathrm{ZnO}$ nanoparticles.

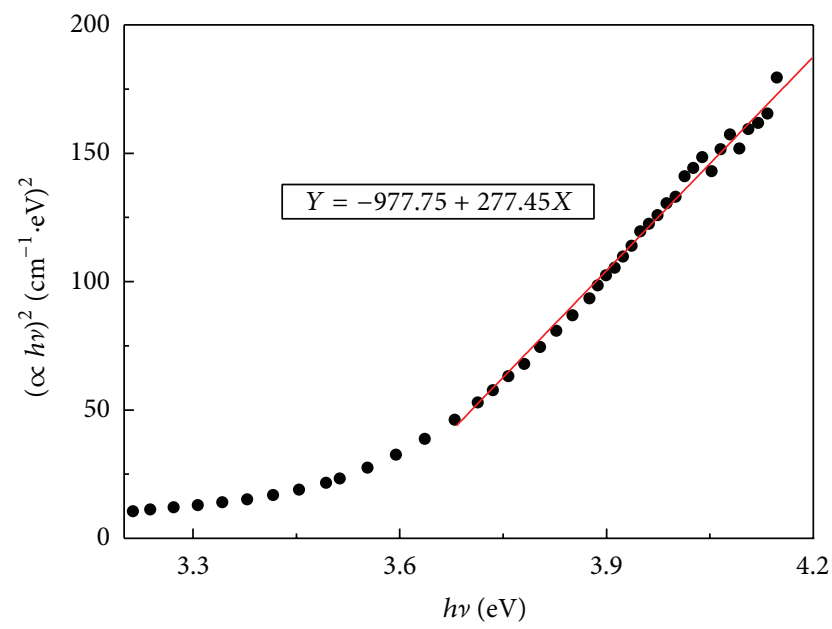

FIGURE 6: $(\alpha h \nu)^{2}$ versus $h \nu$ plot of the synthesized $\mathrm{ZnO}$ nanoparticles. from applying Tauc's equation, is equal to $3.52 \mathrm{eV}$, which is higher than that of the bulk material. Absorption peak of the as-prepared sample is $298 \mathrm{~nm}$ which is highly blue shifted as compared to the bulk $(360 \mathrm{~nm})$. Large optical energy gap and highly blue shifted absorption edge confirm that the prepared $\mathrm{ZnO}$ nanoparticle exhibits strong quantum confinement effect.

\section{Conflict of Interests}

The authors declare that there is no conflict of interests regarding the publication of this paper.

\section{Acknowledgment}

This research was funded by the Deanship of Scientific Research (DSR), King Abdulaziz University, Jeddah, under Grant no. (12/662/1433). The authors, therefore, acknowledge with thanks DSR technical and financial support.

\section{References}

[1] Z. L. Wang and J. Song, "Piezoelectric nanogenerators based on zinc oxide nanowire arrays," Science, vol. 312, no. 5771, pp. 242246, 2006.

[2] D. C. Look, "Recent advances in $\mathrm{ZnO}$ materials and devices," Materials Science and Engineering B, vol. 80, no. 1-3, pp. 383387, 2001.

[3] M. Kitano and M. Shiojiri, "Benard convection $\mathrm{ZnO} /$ resin lacquer coating - a new approach to electrostatic dissipative coating," Powder Technology, vol. 93, no. 3, pp. 267-273, 1997.

[4] I. O. Sosa, C. Noguez, and R. G. Barrera, "Optical properties of metal nanoparticles with arbitrary shapes," The Journal of Physical Chemistry B, vol. 107, no. 26, pp. 6269-6275, 2003.

[5] Y. Yang, H. Chen, B. Zhao, and X. Bao, "Size control of ZnO nanoparticles via thermal decomposition of zinc acetate coated on organic additives," Journal of Crystal Growth, vol. 263, no. 1-4, pp. 447-453, 2004.

[6] M. Purica, E. Budianu, E. Rusu, M. Danila, and R. Gavrila, "Optical and structural investigation of $\mathrm{ZnO}$ thin films prepared 
by chemical vapor deposition (CVD)," Thin Solid Films, vol. 403-404, pp. 485-488, 2002.

[7] J.-H. Lee, K.-H. Ko, and B.-O. Park, "Electrical and optical properties of $\mathrm{ZnO}$ transparent conducting films by the sol-gel method," Journal of Crystal Growth, vol. 247, no. 1-2, pp. 119-125, 2003.

[8] R. Ayouchi, D. Leinen, F. Martín, M. Gabas, E. Dalchiele, and J. R. Ramos-Barrado, "Preparation and characterization of transparent $\mathrm{ZnO}$ thin films obtained by spray pyrolysis," Thin Solid Films, vol. 426, no. 1-2, pp. 68-77, 2003.

[9] Z. M. Dang, L. Z. Fan, S. J. Zhao, and C. W. Nan, "Preparation of nanosized $\mathrm{ZnO}$ and dielectric properties of composites filled with nanosized ZnO," Materials Science and Engineering B, vol. 99, no. 1-3, pp. 386-389, 2003.

[10] C. Wang, W. X. Zhang, X. F. Qian, X. M. Zhang, Y. Xie, and Y. T. Qian, "Room temperature chemical route to nanocrystalline PbS semiconductor," Materials Letters, vol. 40, no. 6, pp. 255258, 1999.

[11] Y. Han, Y. Ding, W. Yin, and Z. Ma, "Preparation of homogeneous $\mathrm{ZnO}$ nanoparticles via precipitation-pyrolysis with $\mathrm{Zn}_{5}(\mathrm{CO} 3)_{2}(\mathrm{OH})_{6}$ as precursor," Transactions of Nonferrous Metals Society of China, vol. 16, no. 5, pp. 1205-1212, 2006.

[12] M. Jitianu and D. V. Goia, "Zinc oxide colloids with controlled size, shape, and structure," Journal of Colloid and Interface Science, vol. 309, no. 1, pp. 78-85, 2007.

[13] C. Pacholski, A. Kornowski, and H. Weller, "Self-assembly of $\mathrm{ZnO}$ : from nanodots to nanorods," Angewandte Chemie, vol. 41, no. 7, pp. 1188-1191, 2002.

[14] J. Buha, I. Djerdj, and M. Nieberberger, "Nonaqueous synthesis of nanocrystalline indium oxide and zinc oxide in the oxygenfree solvent acetonitrile," Crystal Growth \& Design, vol. 7, no. 1, pp. 113-116, 2007.

[15] C. Chen, B. Yu, J. Liu, Q. Dai, and Y. Zhu, "Investigation of $\mathrm{ZnO}$ films on $\mathrm{Si}<111>$ substrate grown by low energy $\mathrm{O}^{+}$assisted pulse laser deposited technology," Materials Letters, vol. 61, no. 14-15, pp. 2961-2964, 2007.

[16] V. D. Mote, Y. Purushotham, and B. N. Dole, "WilliamsonHall analysis in estimation of lattice strain in nanometer-sized $\mathrm{ZnO}$ particles," Journal of Theoretical and Applied Physics, vol. 6, article 6, 2012.

[17] M. Mazhdi and P. Hossein Khani, "Structural characterization of $\mathrm{ZnO}$ and $\mathrm{ZnO}$ : Mn nanoparticles prepared by reverse micelle method," International Journal of Nano Dimension, vol. 2, no. 4, pp. 233-240, 2012.

[18] M. S. Samuel, L. Bose, and K. C. George, "Optical properties of Zno nanoparticles," Academic Review, vol. 16, no. 1-2, pp. 57-65, 2009. 

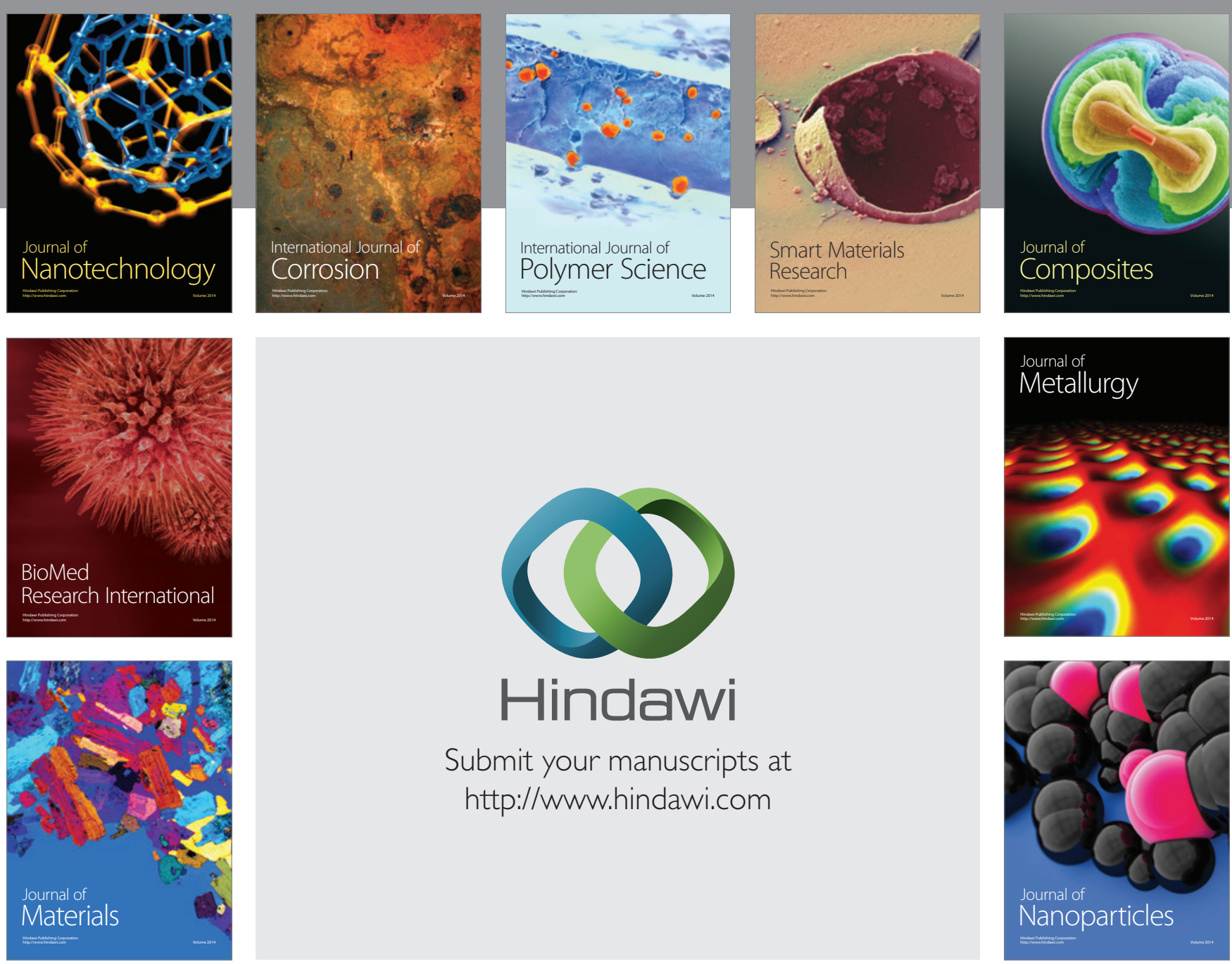

Submit your manuscripts at http://www.hindawi.com
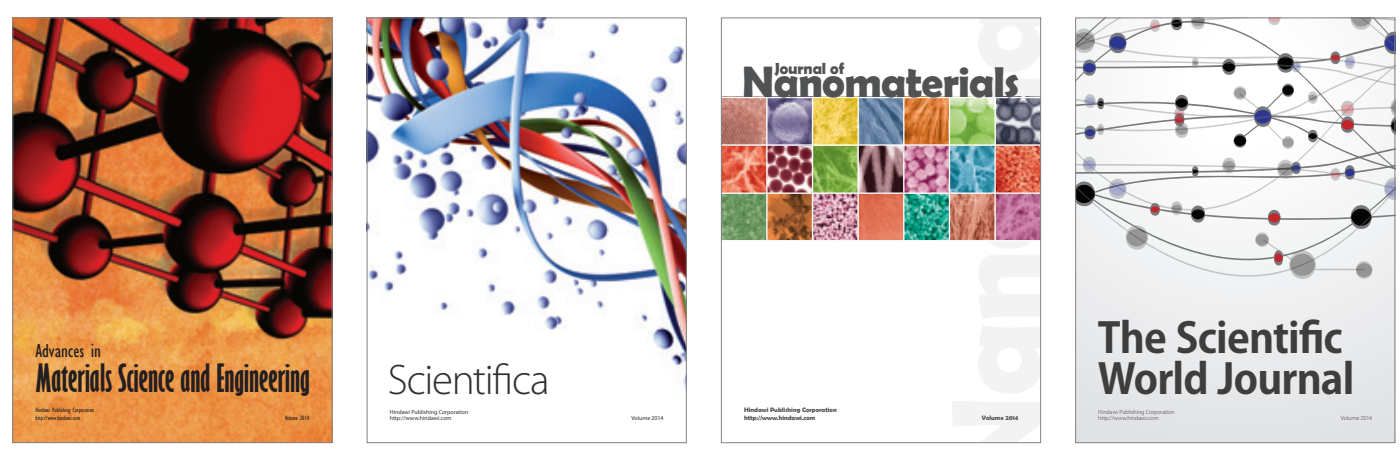

\section{The Scientific World Journal}
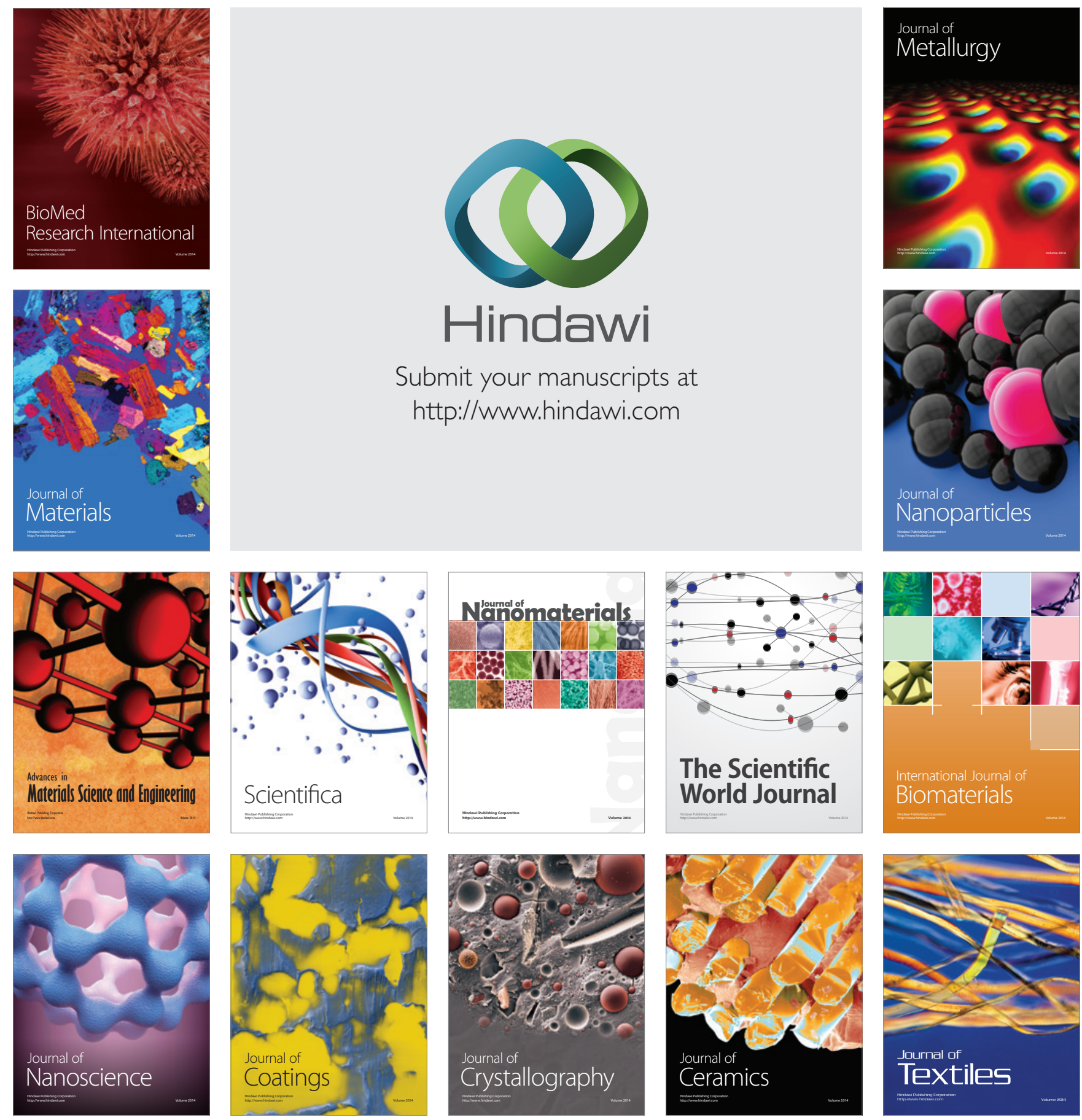